\title{
CONSPECTVS POETARVM
}

L. ACCIVS

Praetextatae

Tragica

Varia

L. AFRANIVS

Togatae

ANONYMI VERSVS

APRISSIVS

Atellana

AQVILIVS

Comica

ATILIVS

Comicum

STATIVS CAECILIVS

Comica

CARMINA VETVSTISSIMA

COMICI INCERTI

Q. ENNIVS

Annales
Comica
Epicharmus
Hedyphagetica
Praetextatae
Saturae
Scenica incerta
Tragica
Varia
PIGRAMMA
Naevii
Pacuvii
Plauti

A. FVRIVS ANTIAS Poemata

HOSTIVS

Bellum Histricum

IVVENTIVS

Comica

D. LABERIVS

Mimi

LICINIVS IMBREX

Comicum
L. LIVIVS ANDRONICVS

86

75

86

Comica

Odyssia

Tragica

C. LVCILIVS

Saturae

95

I64

Q. LVTATIVS CATVLVS

Epigrammata

156 CN. NAEVIVS

66

Bellum Poenicum

Comica

Praetextatae

Tragica

NOVIVS

Atellanae

M. PACVVIVS

Praetextatae

Tragica

POMPILIVS

17 Epigrammata

46 Tragicum

49

L. POMPONIVS

49

46

47

46

32

$4^{8}$

Atellanae

PORCIVS LICINVS

PVBLILIVS SYRVS

Mimi

Sententiae

T. QVINCTIVS ATTA

Togatae

SATVRNII

$\begin{array}{rr}58 & \text { TITINIVS } \\ 164 & \text { Togatae }\end{array}$

TRABEA

I 46

Comica

TRAGICI INCERTI

146 SEX. TVRPILIVS

Comica

66 VALERIVS AEDITVVS

Epigrammata

156

Q. VALERIVS SORANVS VOLCACIVS SEDIGITVS

De poetis
9

7

102

I 49

9

14

14

I 2

154

$5^{8}$

49

I 49

I 49

I50

147

160

I60

95

92

65

89

67

148

146

$14^{8}$ 


\section{Kleine Texte für Vorlesungen und Übungen}

HERAUSGEGEBEN VON HANS LIETZMANN

\section{Alte Philologie in Auswahl}

\section{Griechische Autoren}

33/34 SUPPLEMENTUM LYRICUM. Neue Bruchstücke von Archilochus, Alcaeus, Sappho, Corinna, Pindar, Bacchylides. Ausgew. u. erkl. von E. Diehl. 3. Aufl. 83 S. r9i7. 2.60

112 SUPPLEMENTUM EURIPIDEUM. Bearb. von $H$. von Arnim. 80 S. 19r3. 2.50

II SUPPLEMENTUM SOPHOCLEUM. Hrsg. von E. Diehl. 33 S. I9I3.

44/46 MENANDRI reliquiae nuper repertac. Hrsg. von S. Sudhaus. 2. Aufl. 103 S. 1914. $\mathbf{3 . 2 5}$

66 DIE FROSCHE DES ARISTOPHANES. Mit ausgewälten antiken scholien. Hrsg. von W. Süß. $90 \mathrm{~S}$. I9I I. 2.90

77 HIPPOCRATIS de aere aquis locis. Mit der alten lateinischen Ubersetzung Hrsg. von G. Gundermann. Neudruck. 50 S. 1929. $\quad 1.70$

82 APOLLONIUS DYSCOLUS: De pronominibus. Pars generalis. Edidit Dr. Paulus Maas. 44 S. r9rr.

89 EURIPIDES MEDEA mit scholien. Hrsg. von Ernst Diehl. II6 S. I9ri.

I.75, geb. 2.75

97 DIODORS ROMISCHE ANNALEN bis 302 a. Chr. samt dem Ineditum Vaticanum. Hrsg. von A. B. Drachmann. $72 \mathrm{~S}$. 1912.

2.25

98 MUSAIOS, HERO UND LEANDROS Mit ausgew. Varianten und Scholien. Hrsg. von A. Ludwich. 54 S. Neudruck. 1929.

2.-

II AUSWAHL AUS DEN ILIASSCHOLIEN zur Einführung in die antike Homerphilologie. Ausgew. u. geordn. von W. Deecke. $92 \mathrm{~S}$. 1912 . 2.90

II 8 ORATORIUM ET RHETORUM GRAECORUM nova fragmenta. Edidit K. Jander. 42 S. r9r3. 1.40

120 HIPPOKRATES UBER AUFGABEN UND PFLICHTEN DES ARZTES in einer Anzahl auserlesener Stellen aus dem Corpus Hippocraticum. Hrsg. von Th. Meyer-Steineg und W. Schonack. 27 S. 1913. -.50

r35 NOVAE COMOEDIAE FRAGMENTA in papyris reperta exceptis Menandreis. Edidit O. Schroeder. $77 \mathrm{~S}$. I9r 5.2 .25

137 VITAE HOMERI ET HESIODI in usum scholarum edidit Udalricus de Wilamowitz-Moellendorff. $58 \mathrm{~S}$. Neudruck. 1929. $\quad 1.80$

138 CRATIPPI hellenicorum fragmenta oxyrhynchia. Scholarum in usum edidit J. H. Lipsius. $35 \mathrm{~S}$. 19r6.

145 CALLIMACHI FRAGMENTA NUPER REPERTA. Edidit R. Pfeiffer. 94 S. r92r. 
I49 DIE KLEINEREN HISTORIKERFRAGMENTE AUF PAPYRUS. Bearb. von Fr. Bilabel. 63 S. 1923.

I5I CELSI ALETHEIS LOGOS. Excussit et restituere conatus est Dr. O. Glöckner. XIV, 72 S. 1924 . 3.-

I 58 GORGIAE HELENA. Recognov. et interpretatus est Ot to I mmisch. VII, 55 S. 1927 .

159 ILIAS HOMERI. (J. B. Rexius 1584). Hrsg. von Richard Newald. $56 \mathrm{~S}$. 1929.

r6o LUCIANI DIALOGI MERETRICII In usum scholarum edidit Carolus Mras. 56 S. 1929.

Griechische Inschriften.

II GRIECH. INSCHRIFTEN Z. GRIECH. STAATENKUNDE. Ausgew. von F. Bleckmann. 79 S. I913. 2.50

22 HISTORISCHE GRIECHISCHE INSCHRIFTEN bis auf Alexander den Großen. Ausgew. u. erkl. von $\mathrm{F}$ Nachmanson. 60 S. 1913.

1.90

I56 HISTORISCHF GRIECHISCHE EPIGRAMME. Hrsg. von Friedr. Freiherrn Hiller v. Gaertringen. 64 S. 1926. 3.40

I 10 HISTORISCHE ATTISCHE INSCHRIFTEN. Ausgew. u. erkl. von E. Nachmanson. 2. Aufl. 82 S. r93r. 2.60

Lateinische Inschriften.

29/30 RES GESTAE DIVI AUGUSTI. Das Monumentum Ancyranum. Hrsg. u. erkl. von E. Diehl. 4. Aufl. 52 S. Neudruck. I930. 2 38/40 ALTLATEINISCHE INSCHRIFTEN. Hrsg. von E. Diehl. 3. Aufl. I24 S. 1930 . 4.80 56 POMPEIANISCHE WANDINSCHRIFTEN UND VERWANDTES. Ausgew, von E. Diehl. 2. Aufl, $60 \mathrm{~S}, 193 \mathrm{I}, 3.40$ 62 VULGÄRLATEINISCHE INSCHRIFTEN. Hrsg. von E. Diehl. 180 S. rgro.

68 LATEINISCHE SACRALINSCHRIFTEN Ausgew F. Richter. 45 S. I9II.

Italische Inschrifted.

57 ALTITALISCHE INSCHRIFTEN. Ausgew. von Hermann Jacobsohn. Neudruck. r927. I.--

\section{Papyri and Verwandtes.}

20 ANTIKE FLUCHTAFELN. Hrsg. u. erkl, von Rich. Wünsch. 2. Aufl. 3r S. I9I2.

$-.50$

65 AUS DER ANTIKEN SCHULE. Sammlung griechischer texte auf papyrus, holztafeln, ostraka. Ausgew. u. erkl. von E. Ziebarth. 2. Aufl. $33 \mathrm{~S}$. 19r3.

84 AUS EINEM GRIECHISCHEN ZAUBERPAPYRUS. Von Richard Wünsch. 3r S. I9II. I.-

133 LAMELLAE AVREALE ORPHICAE. Edidit commentario instruxit Alex. Olivieri. $28 \mathrm{~S}$. 1915. -.90

Für die Hefte, deren Verkaufspreis unter RM 2.- liegt, ermäßigt sich der Preis bei Abnahme von 10 und mehr Exemplaren um ro Prozent, bei Abnahme von 25 und mehr Exemplaren um 20 Prozent. 


\section{Grammatik der Griechischen Papyri aus der Ptolemäerzeit}

mit Einschluß der gleichzeitigen Ostraka und der in Ägypten verfaßten Inschriften. Von Professor Dr. EDWIN MAYSER.

Band I: Laut- und Wortlehre. Neue Ausgabe. XIV, 538 Seiten. 1923.

RM I8.-, geb. 19.50

Band II: Satzlehre. Analytischer Teil.

I. Hälfte. XX, 390Seiten. I926. RM 45.- geb.46.50

2. Hälfte. XV,629 Seiten. I934. RM 80.-, geb. 82.-

3. Satzlehre. Synthetischer Teil. VI, 265 S. 1934.

Prospekt auf Wunsch.

RM 36.-, geb. $37 \cdot 50$

\section{Historische griechische Grammatik}

Von Prof. Dr. E. KIECKERS. Vier Bände 1925/26. (Samml. Göschen, Bd. I I 7/18, 924/25). Geb. je RM I.62

\section{Auswahl}

\section{aus griechischen Papyri}

Von R. HELBING. Zweite, veränderte Auflage. I32 S. 1924. (Sammlung Göschen, Bd. 625). Geb. RM I.62

Walter de Gruyter \& Co., Berlin W 10

Genthiner Straße 38 


\section{Hans Lietzman!s \\ Gefchichte Der Alten Kirche}

r. Die Anfänge. Oktav. VIII, 326 Seiten. 2. Auflage. I937. Geb. RM 4.80

2. Ecclesia Catholica. Oktav. VIII, 339 Seiten. I936. Geb. RM 4.80

3. Die Reichskirche. Erscheint Herbst r938.

Jeder Band ist in sich abgeschlossen und einzeln käuflich.

„,...Die meisterhafte Darstellung ist so gestaliet, daß sie auch ohne alle Vorkenntnisse gelesen und verstanden werden kann und doch auch dem Sachkenner neue Perspektiven aufweist und in dem gewissenhaft nachgewiesenen religionsgeschichtlichen und archäologischen Material sowie in der Betrachtung und Auswertung der christlichen Quellen eine Fülle neuer Erkenntnisse und Belehrungen vermittelt."

Christentum und Wissenschaft, Nr. 5, Mai 1935.

\section{Grammatik Der Griechifchen Papyri aus Der Ptolemäerzeit}

mit Einschluß der gleichzeitigen Ostraka und der in Ägypten verfaßten Inschriften. Von Professor Dr. Edwin Mayser. Band I: Laut- und Wortlehre. Neue Ausgabe. XIV, 538 Seiten. 1923. RM I8.-, in Ganzleinen geb. I9.50

Band II: Satzlehre. Analytischer Teil. I. Hälfte. XX, 390 Seiten. I926. RM 45--, in Ganzleinen geb. 46.50 2. Hälfte XV, 629 Seiten. I934. RM 80.-, in Ganzleinen geb. 82.-, 3. Satzlehre. Synthetischer Teil. VI, 265 Seiten. I934. RM 36.-, in Ganzleinen geb. 37.5०

$$
\text { Prospekta } u f W u n s c h
$$

WALTER DE GRUYTER \& CO BERLIN W 35 


